\title{
The THS experiment: Simulating Titan's atmospheric chemistry at low temperature $(200 \mathrm{~K})$
}

\author{
Ella Sciamma-O'Brien ${ }^{1,2}$ Kathleen T. Upton ${ }^{3}$, Jack L. Beauchamp ${ }^{3}$ \\ and Farid Salama ${ }^{1}$ \\ ${ }^{1}$ NASA Ames Research Center, Moffett Field, CA \\ email: ella.m.sciammaobrien@nasa.gov \\ ${ }^{2}$ Bay Area Environmental Research Institute, Petaluma, CA \\ ${ }^{3}$ Noyes Laboratory of Chemical Physics and the Beckman Institute - Caltech, Pasadena, CA
}

\begin{abstract}
In the Titan Haze Simulation (THS) experiment, Titan's atmospheric chemistry is simulated by plasma discharge in the stream of a supersonic expansion, i.e. at low Titan-like temperature $(150 \mathrm{~K})$. Here, we present complementary gas and solid phase analyses of four $\mathrm{N}_{2}-\mathrm{CH}_{4}$-based gas mixtures that demonstrate the unique capability of the THS to monitor the chemical growth evolution in order to better understand Titan's chemistry and the origin of aerosol formation.
\end{abstract}

Keywords. astrochemistry, molecular processes, plasmas, methods: laboratory, planets and satellites: Titan.

\section{Introduction}

Titan, Saturn's largest moon, is the only solid body in the outer solar system with a dense atmosphere. In Titan's atmosphere, composed mainly of nitrogen $\left(\mathrm{N}_{2}\right.$ at 95-98\%) and methane $\left(\mathrm{CH}_{4}\right.$ at 2-5\%), a complex chemistry occurs at temperatures lower than $200 \mathrm{~K}$, and leads to the production of heavy organic molecules and subsequently solid aerosols that form the orange haze surrounding Titan. Because the reactive carbon and nitrogen species present in Titan's aerosols could meet the functionality requirements for precursors to prebiotics, the study of Titan's aerosol has become a topic of extensive research in the fields of astrobiology and astrochemistry. Experiments have been developed in several laboratories in the past 30 years in order to understand the production processes and composition of these atmospheric aerosols.

\section{The Titan Haze Simulation (THS) experiment}

In the study presented here, we used the Titan Haze Simulation (THS) experiment, an experimental setup developed at the NASA Ames COSmIC simulation facility, to study Titan's atmospheric chemistry at low temperature (Ricketts et al. (2011)). In the THS, the chemistry is simulated by plasma in the stream of a pulsed supersonic jetcooled expansion. With this unique design, the gas is cooled to Titan-like temperature $(\sim 150 \mathrm{~K})$ before inducing the chemistry by pulsed plasma (Biennier et al. 2006), and remains at low temperature in the plasma discharge $(\sim 200 \mathrm{~K})$. The pulsed nature of the plasma allows for a truncated chemistry that can be used to study the early stages of aerosol production, which has not been readily accomplished so far using other laboratory production methods. Different $\mathrm{N}_{2}-\mathrm{CH}_{4}$-based gas mixtures can be injected in the plasma. Due to the short residence time of the gas in the pulsed plasma discharge, only the first 


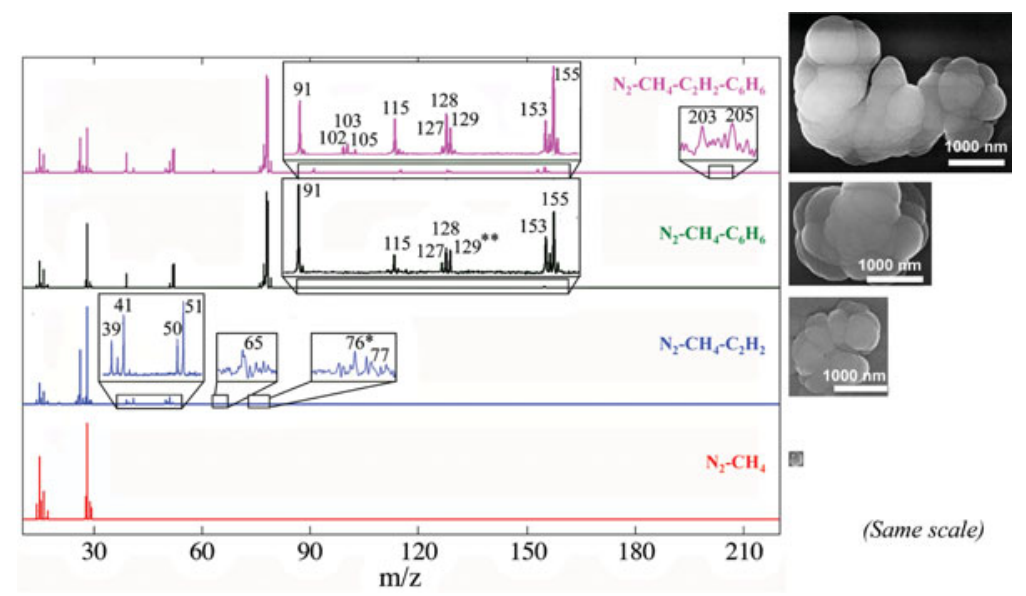

Figure 1. (Left): Mass spectra of four gas mixtures showing the observed chemical growth evolution when adding heavier precursors to the initial $\mathrm{N}_{2}-\mathrm{CH}_{4}$ gas mixture: $\mathrm{N}_{2}-\mathrm{CH}_{4}$ (red), $\mathrm{N}_{2}-\mathrm{CH}_{4}-\mathrm{C}_{2} \mathrm{H}_{2}$ (blue), $\mathrm{N}_{2}-\mathrm{CH}_{4}-\mathrm{C}_{6} \mathrm{H}_{6}$ (green), $\mathrm{N}_{2}-\mathrm{CH}_{4}-\mathrm{C}_{2} \mathrm{H}_{2}-\mathrm{C}_{6} \mathrm{H}_{6}$ (pink). (Right): SEM images of grains and aggregates produced in the same gas mixtures, demonstrating the consistency between gas and solid phase in COSmIC/THS.

steps of the chemistry have time to occur in a $\mathrm{N}_{2}-\mathrm{CH}_{4}$ discharge. However, by adding heavier precursors present as trace elements on Titan to the initial $\mathrm{N}_{2}-\mathrm{CH}_{4}$ mixture, we can observe a chemical growth evolution and study the intermediate steps of Titan's atmospheric chemistry. Both the gas- and solid phase products resulting from the plasmainduced chemistry can be monitored and analyzed using a combination of complementary in situ and ex situ diagnostics.

\section{Results and Discussion}

A recent Time-Of-Flight mass spectrometry study of the gas phase (Sciamma-O'Brien et al. 2014) has demonstrated that the COSmIC/THS is a unique tool to probe the first and intermediate steps of Titan's atmospheric chemistry at Titan-like temperature. In particular, the mass spectra obtained in a $\mathrm{N}_{2}-\mathrm{CH}_{4}-\mathrm{C}_{2} \mathrm{H}_{2}-\mathrm{C}_{6} \mathrm{H}_{6}$ mixture are relevant for comparison to the Cassini Plasma Spectrometer - Ion Beam Spectrometer (CAPS-IBS) instrument. The results of a complementary study of the solid phase are consistent with the chemical growth evolution observed in the gas phase, as shown in Fig. 1. Grains and aggregates form in the gas phase and can be jet deposited on various substrates for ex situ analysis. Scanning Electron Microscopy (SEM) images have shown that aggregates produced in more complex mixtures like $\mathrm{N}_{2}-\mathrm{CH}_{4}-\mathrm{C}_{2} \mathrm{H}_{2}-\mathrm{C}_{6} \mathrm{H}_{6}$ are much larger (up to 5 $\mu \mathrm{m}$ in diameter) than those produced in simpler mixtures like $\mathrm{N}_{2}-\mathrm{CH}_{4}(0.1-0.5 \mu \mathrm{m})$. Direct Analysis in Real Time mass spectrometry (DART-MS) combined with Collision Induced Dissociation (CID) of the solid phase have detected the presence of aminoacetonitrile, a precursor of glycine, in the COSmIC/THS aerosols. X-ray Absorption Near Edge Structure (XANES) measurements also show the presence of imine and nitrile functional groups, showing evidence of nitrogen chemistry. These complementary studies show the high potential of THS to better understand Titan's chemistry and the origin of aerosol formation.

\section{References}

Biennier, L., Benidar, A., \& Salama, F. 2006, Chem. Phys., 326, 445

Ricketts, C. L., Contreras, S. C. Walker, R., \& Salama, F. 2011, Int. J. Mass Spec., 300, 26

Sciamma-O'Brien, E., Ricketts, C. L., \& Salama, F. 2014, Icarus, 243, 325 